\title{
Femtosecond laser induced nanostructuring for surface enhanced Raman spectroscopy
}

Messaoudi, H., Das, S., Lange, J., Heinrich, F., Schrader, S., et al.

H. Messaoudi, S. K. Das, J. Lange, F. Heinrich, S. Schrader, M. Frohme, R. Grunwald, "Femtosecond laser induced nanostructuring for surface enhanced Raman spectroscopy," Proc. SPIE 8972, Frontiers in Ultrafast Optics: Biomedical, Scientific, and Industrial Applications XIV, 89720H (7 March 2014); doi: 10.1117/12.2037052

SPIE. Event: SPIE LASE, 2014, San Francisco, California, United States 


\title{
Femtosecond-laser induced nanostructuring for surface enhanced Raman spectroscopy
}

\author{
H. Messaoudi ${ }^{1,2,3}$, S. K. Das ${ }^{1}$, J. Lange ${ }^{2}$, F. Heinrich ${ }^{2}$, S. Schrader ${ }^{2}$, M. Frohme ${ }^{2}$, and R. Grunwald ${ }^{1} *$ \\ ${ }^{1}$ Max-Born-Institut für Nichtlineare Optik und Kurzzeitspektroskopie, Max-Born-Strasse 2a, \\ D-12489 Berlin, Germany \\ ${ }^{2}$ University of Applied Sciences Wildau, Engineering Department, Bahnhofstrasse, D-15745 \\ Wildau, Germany \\ ${ }^{3}$ Bremen Institute of Applied Beam Technology, Klagenfurter Strasse 2, \\ Bremen, Germany (present address)
}

\begin{abstract}
The formation of periodical nanostructures with femtosecond laser pulses was used to create highly efficient substrates for surface-enhanced Raman spectroscopy (SERS). We report about the structuring of silver and copper substrates and their application to the SERS of DNA (herring sperm) and protein molecules (egg albumen). The maximum enhancement factors were found on $\mathrm{Ag}$ substrates processed with the second harmonic generation (SHG) of a $1-\mathrm{kHz}$ Ti:sapphire laser and structure periods near the SHG wavelength. In the case of copper, however, the highest enhancement was obtained with long-period ripples induced with at fundamental wavelength. This is explained by an additional significant influence of nanoparticles on the surface. Nanostructured areas in the range of $1.25 \mathrm{~mm}^{2}$ were obtained in $10 \mathrm{~s}$. The surfaces were characterized by scanning electron microscopy, Fast Fourier Transform and Raman spectroscopy. Moreover, the role of the chemical modification of the metal structures is addressed. Thin oxide layers resulting from working in atmosphere which improve the biocompatibility were indicated by vibration spectra. It is expected that the detailed study of the mechanisms of laser-induced nanostructure formation will stimulate further applications of functionalized surfaces like photocatalysis, selective chemistry and nano-biology.
\end{abstract}

Keywords: Surface enhanced Raman spectroscopy, laser-induced periodic surface structures, metal substrates, nanostructures, biosensing, DNA detection, protein detection, herring sperm, egg albumen, oxide layers, biocompatibility

\section{INTRODUCTION}

Surface enhanced Raman spectroscopy (SERS) is a highly sensitive technique for the detection of molecules via an enhanced coupling of optical transitions to vibrational modes which is able to work even on single molecule level [1-6]. The efficiency enhancement is obtained by nanoscale features at metal surfaces (field enhancement) as well as the specific charge-transfer between metal and molecule (chemical enhancement). Further advantages of the technique are that it is label free, applicable in real time and that typical substrate materials like $\mathrm{Au}$ and $\mathrm{Ag}$ are corrosion resistant and biocompatible (e.g. if silver is coated with a thin oxygen layer). This makes it an important tool in biology and biomedicine, e.g. for the identification and classification of pathogenic organisms and bio-molecules [7-13] thus enabling for a reliable indication of various diseases. The SERS effect was demonstrated for a variety of different metallic substrates containing metal island films, aggregated metal colloids, electrochemically roughened electrodes, or gold [14] and silver colloidal monolayers. Such substrate structures are either complex and irregular. Because of the high sensitivity of SERS against shape, size, depth and density of the nanoscale features, it is difficult to perform reproducible SERS measurements with random, non-uniform or unstable substrates. Therefore, deterministic patterning strategies such electron beam lithography, nano-imprint lithography, or soft lithography, have been proposed to meet the high standards of bio-medical applications [15,16]. Most of the methods for the generation of regular geometries, however, are time consuming and expensive because of the necessary complex multiple-step procedures and/or the

*grunwald@mbi-berlin.de

Frontiers in Ultrafast Optics: Biomedical, Scientific, and Industrial Applications XIV, edited by Alexander Heisterkamp,

Peter R. Herman, Michel Meunier, Stefan Nolte, Proc. of SPIE Vol. 8972, 89720H

(C) 2014 SPIE · CCC code: 0277-786X/14/\$18 - doi: 10.1117/12.2037052

Proc. of SPIE Vol. $897289720 \mathrm{H}-1$ 
demand for time consuming and expensive because of the necessary complex multiple-step procedures and/or the demand for sophisticated facilities like clean-rooms with chemical and thermal process control. A promising, simple and convenient alternative approach to fabricate patterns of relatively good uniformity and regularity in a fast, cost-effective top-down process is the generation of laser-induced periodic nanostructures (LIPNS) via plasmon interference at the surface of appropriate materials [17]. Here we report on the generation of sub-wavelength scale gratings in $\mathrm{Ag}$ and $\mathrm{Cu}$ with a mode-locked Ti:sapphire laser at two different wavelengths $(800 \mathrm{~nm}, 400 \mathrm{~nm})$. The capability of the nanostructures as efficient substrates for the specific detection of biological matter with SERS is demonstrated for selected complex standard molecules (herring sperm [18], egg albumen protein [19]). Furthermore, the assumption of chemical changes of the substrate surfaces during the laser processing by oxidation in air environment which is interesting for the improvement of biocompatibility is verified by Raman spectra.

\section{NANOSTRUCTURING OF METAL SUBSTRATES WITH ULTRASHORT PULSES}

As it was reported in literature, metallic gratings with periods in few-hundred-nanometer range can be used for efficient SERS [20,21]. The application of intense, ultrashort-pulsed lasers, in particular femtosecond Ti:sapphire lasers, enables to work in a non-thermal ablation regime which is difficult to realize at longer pulse durations where thermal diffusion and melting dominate. The excitation of surface plasmons at the surface, the subsequent photo-induced avalanche ionization and Coulomb explosion are considered to be the basic mechanism of nanostructuring under these conditions $[22,23]$. It has to be mentioned that similar physical mechanisms are also known for dielectric materials and semiconductors where a transient metallic state can be induced by multiphoton absorption. By adjusting the laser parameters like wavelength, polarization, duration and number of pulses and/or fluence, the shape, period and orientation of the created nanostructures can be controlled very precisely [24]. This makes the formation of LIPNS attractive not only for SERS but also many other applications, e.g. for efficient photovoltaic cells [25], LEDs [26], optical memories [27] or hydrophobic functionalized surfaces [28]. Most of the previous SERS activities with LIPNS were performed with non-biological molecules, like Rhodamin6G or benzenethiol. In contrast to other types of nanostructures (e.g. nanoparticles), LIPNS-based substrates $[29,30]$ were reported to exhibit a higher robustness and yield maximum enhancement factors of even more than $10^{9}$ [31]. Furthermore, LIPNS technologies support the miniaturization, e.g. by directly integrating nano-gratings into SERS microchips [32] or micro-fluidic systems [33].

Here we report on SERS studies with biomedical standard samples on fs-laser processed substrates containing LIPNS. The structures were generated by a large-area one-step scanning method. In the application experiments, the biological objects of interest were DNA (herring sperm) and protein (egg-albumen). To evaluate the enhancement, the results are compared to Raman studies with non-structured $\mathrm{Si}$ and glass substrates. The influence of the processing parameters on the SERS activity is discussed. The high observed SERS efficiencies indicate that the LIPNS substrates are well capable to be applied to the emerging field of sensitive molecule detection in biomedicine and neighboring fields.

\section{EXPERIMENTAL TECHNIQUES}

\subsection{Nanostructuring of the substrates}

The structuring method was originally developed for the fabrication of coherently linked LIPNS with a high degree of order on extended areas of $\mathrm{TiO}_{2}$ surfaces in air [34]. In contrast to conventional scanning methods, a substrate is translated through a line focus of an amplified femtosecond Ti:sapphire laser. This enables to enhance the processing speed considerably. The limiting factors are the pulse energy (because the energy has to exceed the ablation threshold all along the focal line) and the intensity profile (the line focus has to be as homogeneous as possible because of the intensity dependence of the plasmonic mechanism of LIPNS formation). If the nanoripples are formed along a line instead of at a point-like focus, the approach is much more efficient compared to conventional techniques. For the fabrication of SERS substrates it was extended to metallic surfaces for the first time, to the best of our knowledge. Two important differences had to be taken into account: (1) In the case of dielectric substrates and an excitation at near infrared wavelengths, typically a transient metallic state corresponding to an enhanced reflectivity appears after multiphoton absorption by generating a plasma state (free carriers). In the case of metals, however, the metallic state and high reflection exist from the beginning and the coupling of energy into the surface is more difficult, and (2) dielectric metal oxides tend to undergo a chemical reduction to metals as a result of fs-laser illumination. Nanostructuring of 
metals in air environment, vice versa, rather tends to end up with an oxidation of the surfaces. Both restrictions are relevant but can also be exploited to specifically govern the complex excitation and reaction dynamics.

In the experiments, an amplified, linearly polarized Ti:sapphire laser (Spitfire, Spectra Physics) with a FWHM pulse duration of $120 \mathrm{fs}$ and a repetition frequency of $1 \mathrm{kHz}$ was used to generate the LIPNS. Apart of the fundamental wavelength $(800 \mathrm{~nm})$, samples were also irradiated at the wavelength of second harmonic generation (SHG) $(400 \mathrm{~nm})$. SHG was provided by a beta-barium-borate $(\mathrm{BBO})$ crystal. The metal substrates $(\mathrm{Ag}, \mathrm{Cu})$ were mounted on a computer controlled x-y-translation stage (MT 65, Newport, velocity range $0.001-2.5 \mathrm{~mm} \mathrm{~s}^{-1}$ ) and moved along the focal plane of a thin cylindrical lens (focal length $f=100 \mathrm{~mm}$ ). The Ag substrates (Good fellows Cambridge Ltd.) had a thickness of $0.5 \mathrm{~mm}$ and a purity of $99.95 \%$. The surface of the substrate was illuminated at normal incidence. The pulse energy was varied from 75 to $260 \mu \mathrm{J}$ at $\lambda_{\text {exc }}=800 \mathrm{~nm}$ and from 30 to $368 \mu \mathrm{J}$ at $\lambda_{\text {exc }}=400 \mathrm{~nm}$, while the translation speed was changed in three steps $(0.005,0.05$ and $0.5 \mathrm{~mm} / \mathrm{s})$. The generated LIPNS were characterized with a field emission scanning electron microscope (FE-SEM, JEOL JSM 64-00F). The spatial frequencies of the nanostructures were quantitatively analyzed by performing a two-dimensional Fast Fourier Transform (FFT) of the SEM image data.

\subsection{Surface enhanced spectroscopy}

For the studies to be reported here, two standard types of bio-molecules were used: (i) DNA (herring sperm) and (b) a protein (egg albumen). These reference substances were obtained from Sigma-Aldrich Co. LLC. $22.8 \mathrm{mg}$ herring sperm $D N A$ were dissolved in a reaction vessel with $1.5 \mathrm{ml}$ of distilled water. Then, $8 \mu \mathrm{l}$ of this solution was pipetted onto the SERS substrates and dried in vacuum for about $5 \mathrm{~min}$. In a similar manner, $51.2 \mathrm{mg}$ egg albumen were dissolved in 500 $\mu 1$ of distilled water. Subsequently, the suspension was briefly stirred with a vortex mixer and set for about 5 minutes in an ultrasonic bath. Eventually, the solution was tipped on the surface of the substrates and dried for 5 min in vacuum.

For the SERS measurements, a commercial confocal micro Raman microscope (WITec alpha 300) was applied. The light source was a $488 \mathrm{~nm}$ DPSS laser the beam of which was coupled to the microscope via a single mode optical fiber, providing a maximum power of about $35 \mathrm{~mW}$ at the surface. A $10 \mathrm{x}$ magnifying objective delivered an optical signal averaging over the surface structures with sufficient statistical confidence. A lens-based spectrograph of $300 \mathrm{~mm}$ focal length (WITec) equipped with a 3-stage Peltier cooled EMCCD detector (DU970N-BV, Andor Technology) served for the spectral analysis. The Raman signal from the focal plane is imaged onto the end face of a multimode fiber of $100 \mu \mathrm{m}$ diameter and transmitted to the spectrometer entrance. Thus, the confocal resolution of the microscope as well as the spectral resolution are determined by the fiber geometry. In order to get the full Raman relevant spectral range, the $600 \mathrm{~g} / \mathrm{mm}$ grating of the spectrometer was employed, resulting in a spectral resolution of approximately $7 \mathrm{~cm}^{-1}$. The laser power was varied from 1 to $5 \mathrm{~mW}$ and the acquisition time of the detection system was fixed at $5 \mathrm{~s}$.

\section{EXPERIMENTAL RESULTS}

\subsection{Laser-induced periodic nanostructures}

At a scanning velocity of $0.5 \mathrm{~mm} / \mathrm{s}$, no distinct periodical nanostructures appeared. The corresponding SERS signals from the irradiated regions were rather comparable to the signals from unstructured regions. Relevant SERS signals, however, were obtained from areas processed at velocities of 0.05 and $0.005 \mathrm{~mm} / \mathrm{s}$. Therefore, the further experiments were exclusively performed at these particular velocities. The line focus method enables a LIPNS formation on a few $\mathrm{mm}^{2}$ even in a single scan so that the procedure comes close to typical parameters for a high spot-to-spot reproducible SERS detection. For example, at a wavelength of $400 \mathrm{~nm}$ and pulse energies between 210 and $360 \mu \mathrm{J}$, the central part of the focal zone containing LIPNS had a lateral dimension of $>2500 \mu \mathrm{m}$. In a complete processing cycle, the substrates were linearly moved over a total distance of $500 \mu \mathrm{m}$ at scanning velocity of $0.05 \mathrm{~mm} / \mathrm{s}$ during a time interval of $10 \mathrm{~s}$. Thus, the resulting nanostructured area was about $500 \times 2500 \mu \mathrm{m}^{2}$ after each particular scanning procedure. Because of the slightly non-uniform intensity profile, the depth profile of the LIPNS was not perfectly constant.

It is important to notice that, beside the ripple-like nanostructures, the formation of distributed nanoparticles with characteristic diameters of some $10 \mathrm{~nm}$ was observed at a scanning velocity of $0.005 \mathrm{~mm} / \mathrm{s}$ and/or at the SHG wavelength of $400 \mathrm{~nm}$.

An overview on the characteristic parameters of the generated LIPNS and micrometer-range random structures in $\mathrm{Ag}$ in dependence on the processing parameters is given in Table 1. 
Table.1: Characteristic parameters of the laser-induced nanostructures on a silver surface depending on the wavelength and scanning speed (fs-laser: amplified Ti:sapphire, $120 \mathrm{fs}, 800 \mathrm{~nm}, 1 \mathrm{kHz}$ ).

\begin{tabular}{|c|c|c|}
\hline$\lambda_{\text {exc }}[\mathrm{nm}]$ & Scan velocity $[\mathrm{mm} / \mathrm{s}]$ & Feature size of structures \\
\hline \multirow{2}{*}{800} & 0.05 & LIPNS, $530 \pm 25 \mathrm{~nm}$ \\
\cline { 2 - 3 } & 0.005 & random, 1 to $3 \mu \mathrm{m}$ \\
\cline { 2 - 3 } & 0.05 & LIPNS, $530 \pm 25 \mathrm{~nm}$ \\
\hline \multirow{2}{*}{400} & \multirow{2}{*}{0.005} & random, 1 to $3 \mu \mathrm{m}$ \\
\cline { 2 - 3 } & & LIPNS $253, \pm 15 \mathrm{~nm}$ \\
\cline { 2 - 3 } & &
\end{tabular}

At a scanning velocity of $0.05 \mathrm{~mm} / \mathrm{s}$, a dominance of low spatial frequency LIPNS was found within pulse energy windows of $150-250 \mu \mathrm{J}$ and $210-360 \mu \mathrm{J}$ at excitation wavelengths of $\lambda_{\mathrm{exc}}=800 \mathrm{~nm}$ and $400 \mathrm{~nm}$, respectively. Typical SEM images of the corresponding LIPNS at pulse energies of $180 \mu \mathrm{J}$ (fundamental wavelength at $800 \mathrm{~nm}$ ) and $330 \mu \mathrm{J}$ (SHG at $400 \mathrm{~nm}$ ) are shown in Fig. 1.

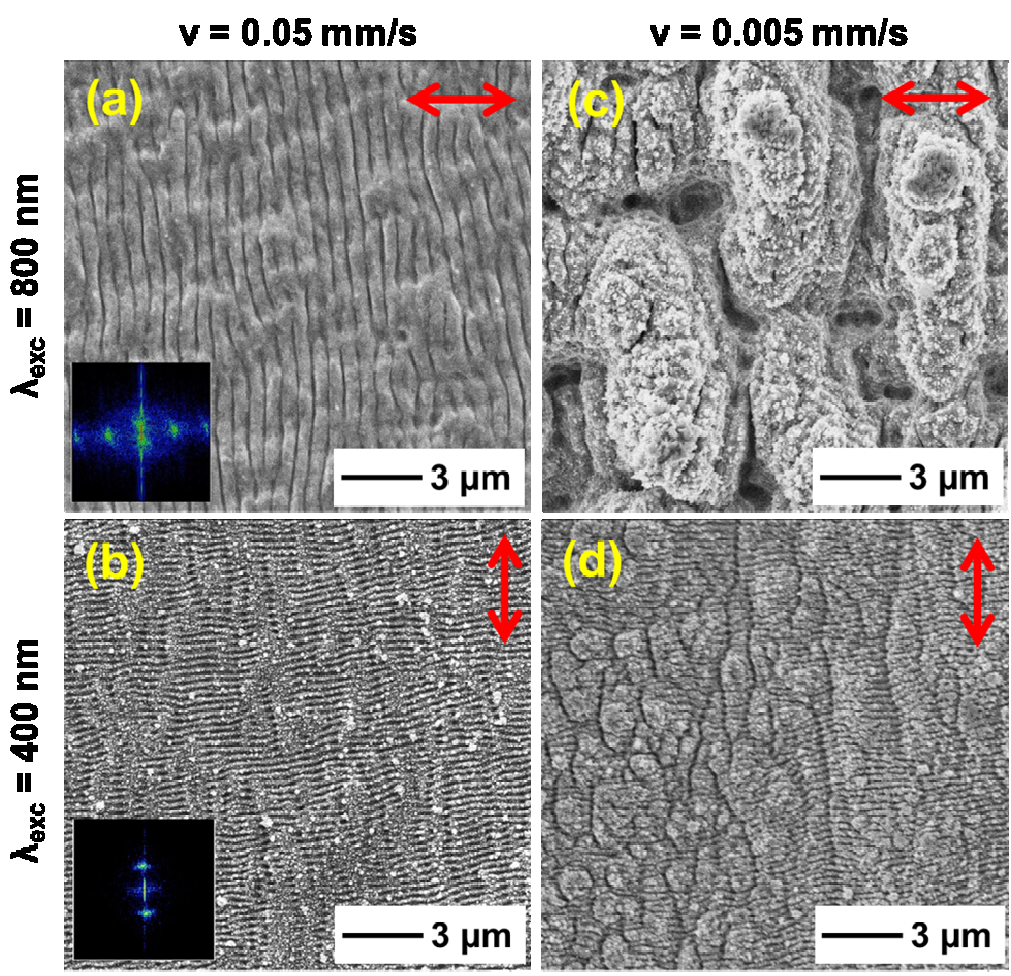

Figure 1 (color online): SEM images of laser-induced periodic surface structures on bulk Ag substrates obtained at different excitation wavelengths and scan velocities. The ripples in (a) and (b) were determined to have periods of $520 \pm 20 \mathrm{~nm}$ and $255 \pm 30 \mathrm{~nm}$, respectively (insets: FFT plots) and can be related to plasmon interference and nonthermal ablation. The pictures in (c), (d) show the appearance of superimposed random structures on fewmicrometer scale indicating an additional influence or even dominance (as in c) of global material transport processes.

The insets of Fig. 1(a) and Fig. 1(b) show 2D Fast Fourier Transform (FFT) plots, indicating the degree of periodicity of the structures. The analysis yields average values of period, $p=530 \mathrm{~nm}$ and $p=255 \mathrm{~nm}$ for fundamental and SHG, respectively. The orientation of the LIPNS was found to be perpendicular to the polarization vector of the input radiation at both fundamental or SHG wavelengths. 


\subsection{SERS measurements}

Measured SERS signals from herring sperm DNA from LIPNS created at fundamental and SHG wavelengths are shown in Fig. 2. The spectra contain several distinct peaks. Some characteristic bands between 600 and $1800 \mathrm{~cm}^{-1}$ can be allocated according to ref. [35]. The two distinct peaks at $\sim 1160$ and $1455 \mathrm{~cm}^{-1}$ can be attributed to deoxyribosephosphate and the peaks at 1232, 1369 and $1509 \mathrm{~cm}^{-1}$ are believed to belong to adenine, while the band around $800 \mathrm{~cm}^{-1}$ can likely be assigned to stretching vibrations in the main chain of an A-Type DNA. The band at $1014 \mathrm{~cm}^{-1}$ - observed only with the $400 \mathrm{~nm}$ LIPNS - can be related to $\mathrm{C}-\mathrm{O}$ stretching vibrations. Moreover, there is a strong evidence for a cytosine band at $623 \mathrm{~cm}^{-1}$ and another deformation mode band of $\mathrm{NH}_{2}$-group in the adenine-guanine-cytosine molecules at $1600 \mathrm{~cm}^{-1}$. The bands indicated at 2900 and $2945 \mathrm{~cm}^{-1}$ can be attributed to some $\mathrm{C}-\mathrm{H}$ stretching vibrations in the $\mathrm{CH}_{2^{-}}$ groups [3].

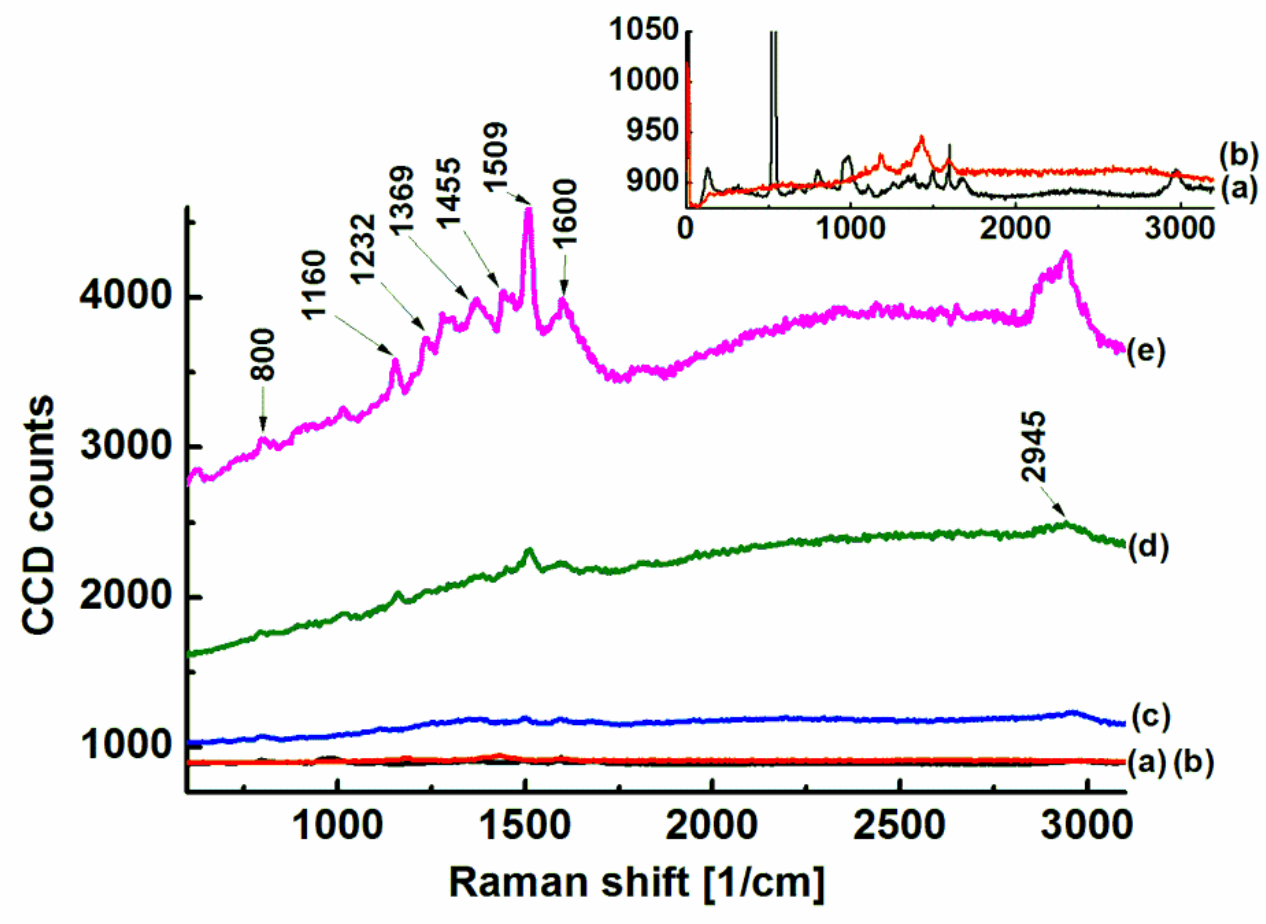

Figure 2 (color online): Detected SERS signals from herring sperm on Ag substrate in comparison to unstructured standard substrates: (a) unstructured $\mathrm{Si}$, (b) unstructured glass, (c) unstructured Ag, (d) Ag with LIPNS (period $530 \mathrm{~nm}$ ), (e) Ag with LIPNS (period $255 \mathrm{~nm}$ ).

The comparison of SERS spectra of egg albumen (Fig. 3) on the Ag substrate with the signals from unstructured Si and glass surfaces proves that the Ag-surface with LIPNS enables to reliably detect the protein bands. The detected peaks were centered at 524, 675, 984, 1355, 1425 and $1614 \mathrm{~cm}^{-1}$. According to the work of Ngarize et al. [19], the band at 524 $\mathrm{cm}^{-1}$ results from a disulphide bond of ovalbumen, which accounts already up to a share $54 \%$ of the egg white. The peaks at 1355 and $1613 \mathrm{~cm}^{-1}$ are probably related to the helical structure and to the Amide1 band in egg albumen [19]. For structures generated at the lowest scan velocity of $\mathrm{v}=0.005 \mathrm{~mm} / \mathrm{s}$, the SERS signals were strongly dependent on the position of measurement on the surface. SERS signals were only detectable when the probe beam was directly focused into the area containing LIPNS.

The SERS spectral fingerprints appear to be almost similar from all LIPNS regions independent on the generation wavelength $(400 \mathrm{~nm}$ or $800 \mathrm{~nm})$, i.e. the periods are not a critical issue for the molecular excitation paths in the range of chosen parameters. The enhancement of the SERS signals, however, was by a factor $>2$ higher within LIPSS regions structured at $400 \mathrm{~nm}$ compared to the substrates structured at $800 \mathrm{~nm}$. This observation confirms the results of Kahl et al. [21], where it was demonstrated that a systematical step-down variation of the period of Ag-coated Si gratings between 800 and $400 \mathrm{~nm}$ enables a scaling of the SERS enhancement factor up to a factor of 2. 


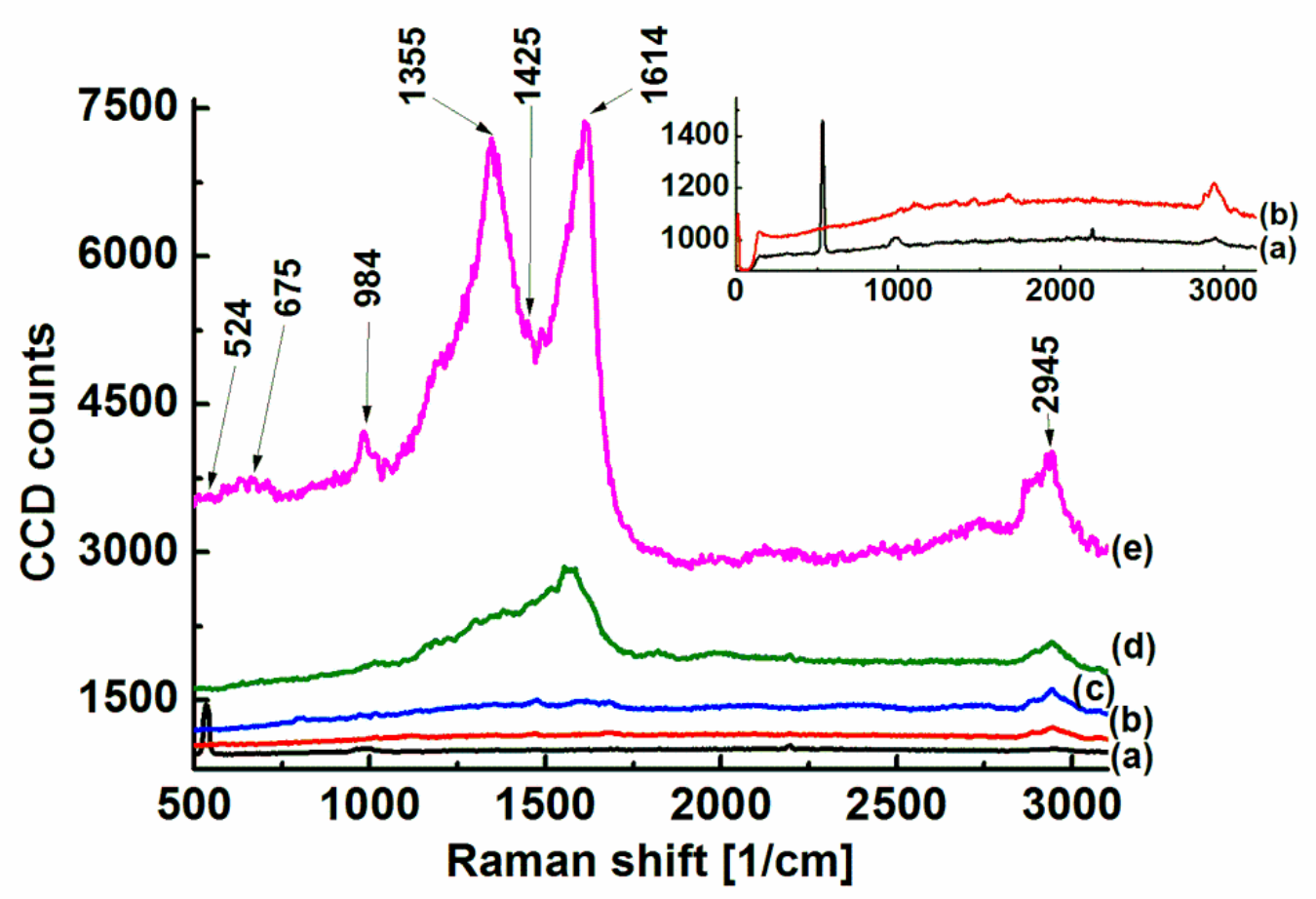

Figure 3 (color online): Detected SERS signals from egg albumen on Ag substrate in comparison to unstructured standard substrates: (a) unstructured $\mathrm{Si}$, (b) unstructured glass, (c) unstructured Ag, (d), Ag with LIPNS (period 530 nm). (e) Ag with LIPNS (period $255 \mathrm{~nm}$ ).

It has to be emphasized that the determination of the exact enhancement factors for SERS on biomedical samples is nontrivial because of its strong dependence on the homogeneity. On the basis of 10 spots for each sample and for $>70 \%$ of the cases we found well detectable Raman signals of DNA and protein with LIPNS on Ag substrates. On the contrary, Raman signals on $\mathrm{Si}$ and glass substrates taken under identical conditions were found to be either undetectable or very weak as it can clearly be recognized in the insets of Fig. 2 and Fig. 3. These results agree with the findings of the group of Chang et. al. for Rhodamine 6G [20]. Compared to the unstructured silver, enhancement factors up to 20 after the femtosecond laser structuring were observed in our experiments. The maximum enhancement factor for DNA and protein with respect to Si and glass substrates was found to be about 200 .

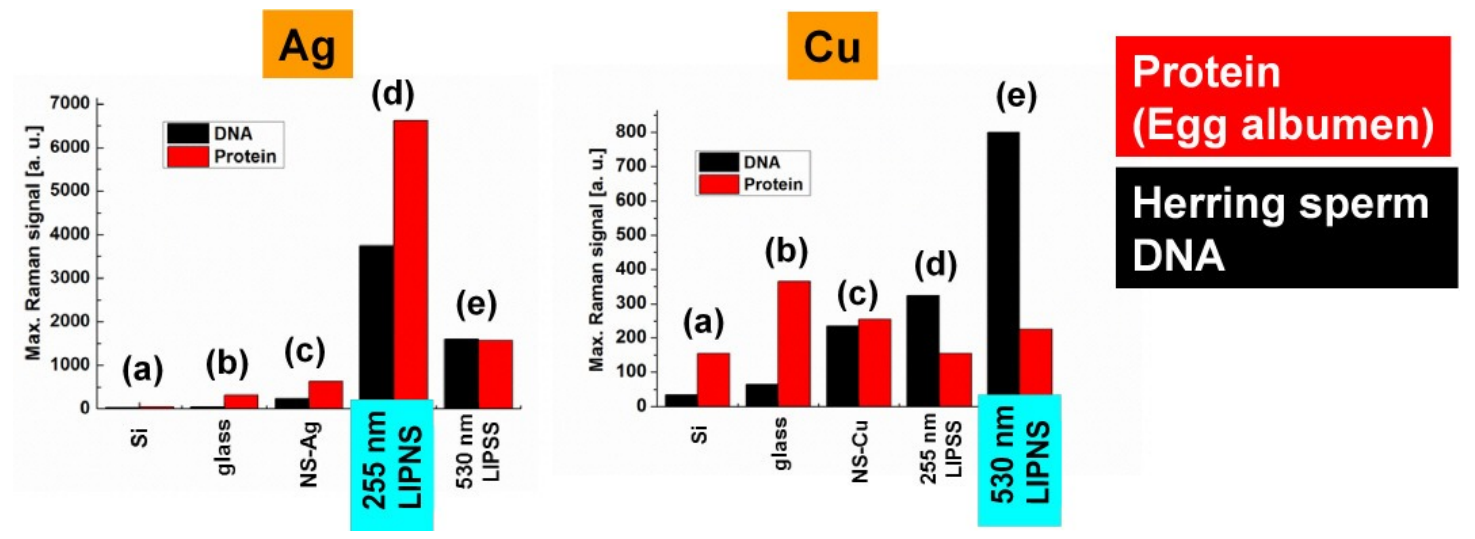

Figure 4 (color online): Comparison of SERS experiments with egg albumin and herring sperm for $\mathrm{Ag}$ (left) and $\mathrm{Cu}$ (right) substrates for the cases of unstructured and structured surfaces: (a) unstructured $\mathrm{Si}$, (b) unstructured glass, (c) unstructured metal, (d), metal with LIPNS (period $255 \mathrm{~nm}$ ), (e) metal with LIPNS (period $530 \mathrm{~nm}$ ). 


\section{CONCLUSIONS}

To conclude, the generation of laser-induced periodic nanostructures (LIPNS) on metals (in particular Ag and $\mathrm{Cu}$ ) for the application as SERS substrates in biomedicine was demonstrated. The structuring of extended areas was realized by translating a substrate through a line focus of an amplified femtosecond Ti:sapphire laser at wavelengths of $800 \mathrm{~nm}$ and $400 \mathrm{~nm}$. The line scan technique makes the fabrication simple, cost-effective and significantly faster compared to conventional scanning systems with circular focal spots and meandering movement. The plasmon-based mechanisms of nanostructure formation in bulk metal samples and chemical changes of the surfaces were studied to optimize the process parameters. It was shown that LIPNS can be obtained in areas as large as $2500 \times 500 \mu \mathrm{m}^{2}$ within a processing time of only $10 \mathrm{~s}$. The capability of the structured substrates for the sensitive detection of selected bio-molecules with surface enhanced Raman spectroscopy (SERS) was successful verified by proof-of-principle experiments with egg albumen protein and herring sperm DNA. SERS under well-defined wetting conditions for the biomolecules to be detected was realized. In case of Ag substrates, the SERS signals for a grating period of $255 \mathrm{~nm}$ exceed the signals for a period of 530 $\mathrm{nm}$ by a factor $>2$, as a consequence of the higher structural density. The relatively high achievable uniformity with respect to period, depth and shape of the LIPNS promises an improved reproducibility of the spectroscopic signals. The data were compared to Raman measurements with reference Si and glass substrates under identical conditions. In both cases, the signals were either undetectable or very weak. Compared to unstructured smooth Ag surfaces, the SERS signals were enhanced by factors up to 200 (typically a factor 20). Future activities have to include a postprocessing of the metal surfaces, the combination of nanoripple generation with the insertion of smaller nanoparticles and time dependent studies of charge transfer processes at nanostructured surfaces.

\section{ACKNOWLEDGMENTS}

The authors thank T. Elsaesser (MBI, Berlin) and W. Seeber (Otto Schott Institute, Jena). The studies were financially supported by DFG (project GR 1782/12-2, UP-LINN).

\section{REFERENCES}

[1] Natan, M. J., "Surface enhanced Raman scattering," Faraday Discuss. 132, 321-328 (2006).

[2] Barhoumi, A., Zhang, D., Tam, F., and Halas, N. J., "Surface-enhanced Raman spectroscopy of DNA," J. Am. Chem. Soc. 130, 5523-5529 (2008).

[3] Taylor, C. E., Garvey, S. D., and Pemberton, J. E., "Carbon contamination at silver surfaces: surface preparation procedures evaluated by Raman spectroscopy and X-ray photoelectron spectroscopy," Anal. Chem. 68, 2401-2408 (1996).

[4] Pahlow, S., März, A., Seise, B., Hartmann, K., Freitag, I., Kämmer, E., Böhme, R., Deckert, V., Weber, K., Cialla, D., Popp, J., "Bioanalytical application of surface- and tip-enhanced Raman spectroscopy," Eng. Life Sci. 12, 131-143 (2012).

[5] Küstner, B., Gellner, M., Schutz, M., Schoppler, F., Marx, A., Ströbel, P., Adam, P., Schmuck, C., and S. Schlücker, "SERS labels for red laser excitation: silica-encapsulated SAMS on tunable gold/silver nanoshells," Angew. Chem., Int. Ed. 48, 19501953 (2009).

[6] Culha, M., Cullum, B., Lavrik, N., and Klutse, C. K., "Surface-enhanced Raman scattering as an emerging characterization and detection technique," J. Nanotechnology 2012, 971380 (2012).

[7] Driskell, J. D., Zhu, Y., Kirkwood, C. D., Zhao, Dluhy, Y., and R. A., Tripp, R. A., "Rapid and sensitive detection of rotavirus molecular signatures using surface enhanced Raman spectroscopy," PLoS ONE 5, e10222 (2010).

[8] Feng, S. Y., Lin, J. Q., Cheng, M., Li, Y. Z., Chen, G. N., Huang, Z. F., Yu, Y., Chen, R., and Zeng, H. S., "Gold nanoparticle based surface-enhanced Raman scattering spectroscopy of cancerous and normal nasopharyngeal tuissues under near-infrared laser excitation," Appl. Spectrosc. 63, 1089-1094 (2009).

[9] He, Y., Su, S., Xu, T., Zhong, Y., Zapien, A., Li, J., Fan, C. and Lee, S.-T., "Silicon nanowires-based highly-efficient SERS-active platform for ultrasensitive DNA detection," Nano Today 6, 122-130 (2011).

[10] Hennigan, S. L., Driskell, J. D., Dluhy, R. A., Zhao, Y., Tripp, R. A., Waites, K. B., and Krause, D. C., "Detection of mycoplasma pneumoniae in simulated and true clinical throat swab specimens by nanorod array-surface-enhanced Raman spectroscopy," PLoS ONE 5, e13633 (2010).

[11] Chen, P., Shen, A. G., Zhao, W., Baek, S. J., Yuan, H., and Hu, J. M., "Raman signature from brain hippocampus could aid Alzheimer's disease diagnosis," Appl. Opt. 48, 4743 (2009).

[12] Shen, J., Fan, L., Yang, J., Shen, A. G., and Hu, J. M., "A longitudinal Raman microspectroscopic study of osteoporosis induced by spinal cord injury," Osteoporosis Int. 21, 81-87 (2010).

[13] David, C., Guillot, N., Shen, H., Toury, T., de la Chapelle, M. L., "SERS detection of biomolecules using lithographed nanoparticles towards a reproducible SERS biosensor," Nanotechnology. 21, 475501-47550 (2010).

[14] Karatas, Ö. F., Sezgin, E., Aydin, Ö., and Culha, M., "Interaction of gold nanoparticles with mitochondria," Colloids Surf. B 71, 315-318 (2009). 
[15] Zheng, W., Chiamori, H. C., Liu, G. L., Lin, L., and Chen, F. F., "Nanofabricated plasmonic nano-bio hybrid structures in biomedical detection, Nanotechnol. Rev. 1, 213-233 (2012).

[16] Xie, W., and Mao, C., "Bio-imaging, detection and analysis by using nanostructures as SERS substrates," J. Mater. Chem. 21, 5190-5202 (2011).

[17] Bonse, J., Rosenfeld, A., and Krüger, J., "On the role of surface plasmon polaritons in the formation of laser-induced periodic surface structures upon irradiation of silicon by femtosecond-laser pulses," J. Appl. Phys. 106, 104910 (2009).

[18] Ke, W., Yu, D., and Wu, J., "Raman spectroscopic study of the influence on herring sperm DNA of heat treatment and ultraviolet radiation," Spectrochimica Acta Pt. A 55, 1081-1090 (1999).

[19] Ngarize, S., Adams, A., Howell, and N. K., "Studies on egg albumen and whey protein interactions by FT-Raman spectroscopy and rheology," Food Hydrocolloids 18, 49-59 (2004).

[20] Chang, H. W., Tsai, Y. C., Cheng, C. W., Lin, C. Y., Lin, Y. W., and Wu, T. M., "Nanostructured Ag surface fabricated by femtosecond laser for surface-enhanced Raman scattering," J. Colloid Interface Sci. 360, 305-308 (2011).

[21] Kahl, M., Voges, E., Kostrewa, S., Viets, C., and Hill, W., "Periodically structured metallic substrates for SERS," Sensors and Actuators B 51, 285-291 (1998).

[22] Vorobyev, A. Y., and Guo, C. "Effects of nanostructure-covered femtosecond laser induced periodic surface structures on optical absorptance of metals," Appl. Phys. A 86, 321-24 (2007).

[23] Shen, M., Carey, J. E., Crouch, C. H., Kandyla, M., Stone, H. A., and Mazur, E., "High-density regular arrays of nanometerscale rods formed on silicon surfaces via femtosecond laser irradiation in water," Nano Letters 8, 2087-2091 (2008).

[24] Taylor, R., Hnatovsky, C., and Simova, E., "Applications of femtosecond laser induced self-organized planar nanocracks inside fused silica glass," Laser \& Photon. Rev. 2, 26-46 (2008).

[25] Torres, R., Itina, T. E., Vervisch, V., Halbwax, M., T. Derrien, Sarnet, T., Sentis, M., Ferreira, J., F. Torregrosa, and L. Roux, "Study on laser induced periodic structures and photovoltaic application," AIP Conf. Proc. 1278, 576-581 (2010).

[26] Chen, J. T., Lai, W. C., Kao, Y. J., Yang, Y. Y., and Sheu, J. K., "Laser-induced periodic structures for light extraction efficiency enhancement of GaN-based light emitting diodes," Opt. Express 20, 5689-5695 (2012).

[27] Shimotsuma, Y., Sakakura, M., Miura, K., Qiu, J. R., Kazansky, P. G., Fujita, K., and Hirao, K., "Application of femtosecondlaser induced nanostructures in optical menory," J. Nanosci. Nanotech. 71, 94104, 2078-0338 (2007).

[28] Baldacchini, T., Carey, J. E., Zhou, M., and Mazur, E., "Superhydrophobic surfaces prepared by microstructuring of silicon using a femtosecond laser," Langmuir 22, 4917-4719 (2006).

[29] Diebold, E. D., Mack, N. H., Doorn, S. K., and Mazur, E., "Femtosecond laser-nanostructured substrates for surface-enhanced Raman scattering," Langmuir 25, 1790-1794 (2009).

[30] Buividas, R., Stoddart, and P. R., Juodkazis, S., "Laser fabricated ripple substrates for surface-enhanced Raman scattering," Ann. Phys. 524, L5-L10 (2012).

[31] Lin, C. H., Jiang, L., Chai, Y. H., Xiao, H., Chen, S. J., and Tsai, H. L., "One-step fabrication of nanostructures by femtosecond laser for surface-enhanced Raman scattering," Opt. Express 17, 21581-21589 (2009).

[32] Lin, C. H., Jiang, L., Xiao, H., Chen, S. J., and Tsai, H. L., "Surface-enhanced Raman scattering microchip fabricated by femtosecond laser," Opt. Lett. 35, 2937-2939 (2010).

[33] Liu, G. L., and Lee, L. P., "Nanowell surface enhanced Raman scattering arrays fabricated by soft-lithography for label-free biomolecular detections in integrated microfluidics," Appl. Phys. Letts. 87, 074101 (2005).

[34] Das, S. K., Dasari, K., Rosenfeld, A., and Grunwald, R., "Extended-area nanostructuring of $\mathrm{TiO}_{2}$ with femtosecond laser pulses at $400 \mathrm{~nm}$ using a line focus," Nanotechnology 21, 155302 (2010).

[35] Ke, W., Yu, D., and Wu, J., "Raman spectroscopic study of the influence on herring sperm DNA of heat tratment and ultraviolet radiation," Spectrochimica Acta Pt. A 55, 1081-1090 (1999). 